Acta Crystallographica Section E

Structure Reports

Online

ISSN 1600-5368

\section{Holger B. Friedrich, ${ }^{a}$ R. Alan Howie $^{b_{*}}$ and Martin O. Onani ${ }^{c}$}

${ }^{\mathbf{a}}$ School of Pure and Applied Chemistry, Howard College, University of KwaZulu-Natal, Durban 4041, South Africa, ' Department of Chemistry, University of Aberdeen, Meston Walk, Aberdeen AB24 3UE, Scotland, and ${ }^{\mathrm{C}}$ Department of Chemistry, University of Transkei, Private Bag X1, UNITRA, 5117 Umtata, South Africa

Correspondence e-mail: r.a.howie@abdn.ac.uk

\section{Key indicators}

Single-crystal X-ray study

$T=295 \mathrm{~K}$

Mean $\sigma(\mathrm{C}-\mathrm{C})=0.007 \AA$

$R$ factor $=0.026$

$w R$ factor $=0.072$

Data-to-parameter ratio $=13.7$

For details of how these key indicators were automatically derived from the article, see http://journals.iucr.org/e.

\title{
Tricarbonyl(3-carboxypropyl) $\left(\eta^{5}\right.$-cyclopenta- dienyl)tungsten(II)
}

The bond lengths and angles in the title compound, [W $\left.\left(\mathrm{C}_{5} \mathrm{H}_{5}\right)\left(\mathrm{C}_{4} \mathrm{H}_{7} \mathrm{O}_{2}\right)(\mathrm{CO})_{3}\right]$, are as expected for a molecule of this kind. The presence of the carboxylic acid group leads, however, to the creation of hydrogen-bonded dimers consisting of pairs of centrosymmetrically related molecules.

\section{Comment}

The determination of the structure of the title compound, (I), was undertaken as part of our ongoing study of the chemistry of heterodinuclear compounds (Friedrich et al., 2004). Fig. 1 is a drawing of the molecule and selected bond lengths and angles are given in Table 1 . In both cases, the participation of the cyclopentadienyl $(\mathrm{Cp})$ group in the coordination of $\mathrm{W}$ is represented, purely for convenience, by the notional bond $\mathrm{W}-C g 1$ where $C g 1$ is the centroid of the five-membered cyclopentadienyl ring. On this basis, $\mathrm{W}$ is effectively fivecoordinate in a distorted square-pyramidal environment with apical $\mathrm{Cp}$. This arrangement creates the appearance of a stool with $\mathrm{Cp}$ as its seat and the three carbonyl groups and the carboxy ligand as its feet, four in number.

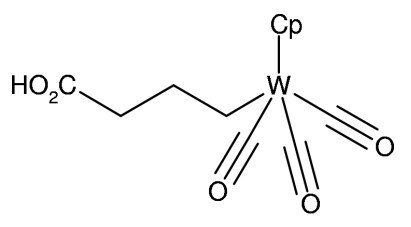

(I)

The bond lengths and angles given in Table 1 are unremarkable. The same is true for the $\mathrm{W}-\mathrm{C}_{\mathrm{Cp}}$ bonds in the range 2.312 (4) -2.376 (4) $\AA$ and the $\mathrm{C}-\mathrm{C}$ bonds and $\mathrm{C}-\mathrm{C}-\mathrm{C}$ angles of $\mathrm{Cp}$ in the ranges 1.385 (7)-1.431 (7) $\AA$ and 107.3 (5)$108.4(5)^{\circ}$, respectively. In the drawing of the unit-cell contents (Fig. 2) a notable feature is the presence of the hydrogenbonded dimer involving a pair of centrosymmetrically related molecules. The hydrogen-bond parameters are given in Table 2. No other intermolecular contacts of any significance, other than van der Waals interactions, are present in the structure. Similar hydrogen-bonded dimers are present in the structures of the analogous compounds $\left[\mathrm{Cp}(\mathrm{CO})_{3} \mathrm{MoCH}_{2} \mathrm{COOH}\right]$ and $\left[\mathrm{Cp}(\mathrm{CO})_{2} \mathrm{FeCH}_{2} \mathrm{COOH}\right]$ (Ariyaratne et al., 1969). Despite the limited quality of the refinements, these authors suggested that there was evidence to support some form of interaction between the metal (Mo or Fe) and the carboxylic acid group. There is no evidence for such an interaction in (I), which would be less likely in any case because of the length of the alkyl chain. There is perhaps a case for redetermining the earlier structures.
Received 4 October 2004 Accepted 8 October 2004 Online 16 October 2004 


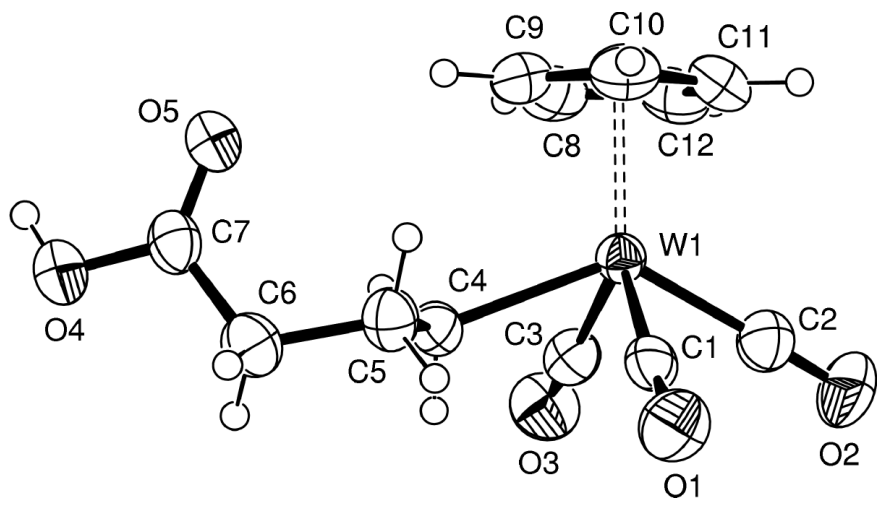

Figure 1

The molecule of (I). Non-H atoms are shown as $50 \%$ probability displacement ellipsoids and $\mathrm{H}$ atoms as small circles of arbitrary radii. The dashed bond joins $\mathrm{W}$ and the centroid of the $\mathrm{Cp}$ ring.

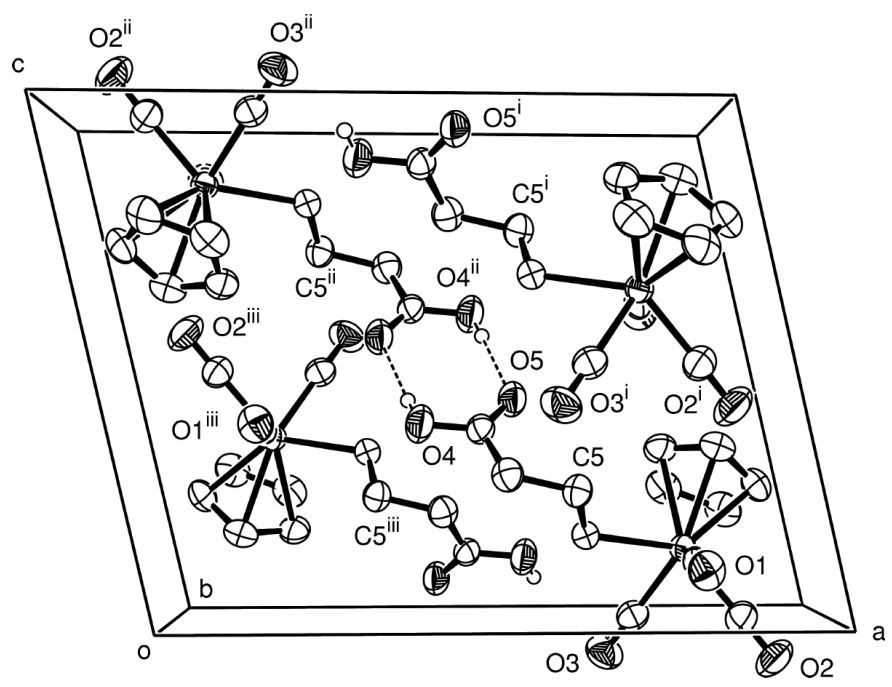

Figure 2

The unit-cell contents of (I). Non-H atoms are shown as $50 \%$ probability displacement ellipsoids and $\mathrm{H}$ atoms as small circles of arbitrary radii. All $\mathrm{H}$ atoms other than those involved in hydrogen bonding (dashed lines) have been omitted. Selected atoms are labelled. [Symmetry codes: (i) $x$, $\frac{1}{2}-y, \frac{1}{2}+z$; (ii) $1-x, 1-y, 1-z$; (iii) $1-x, \frac{1}{2}+y, \frac{1}{2}-z$.]

\section{Experimental}

Compound (I) was obtained by hydrolysis brought about by the presence of water in a dichloromethane/hexane solution of $\left[\mathrm{Cp}(\mathrm{CO})_{3} \mathrm{~W}\left(\mathrm{CH}_{2}\right)_{3} \mathrm{C}(\mathrm{O}) \mathrm{Mo}(\mathrm{CO})\left(\mathrm{PMe}_{3}\right)\left(\mathrm{PPh}_{3}\right) \mathrm{Cp}\right]$ (Onani, 2002). Yellow crystals suitable for analysis were obtained after $5 \mathrm{~d}$ of slow diffusion of hexane into a dichloromethane solution of (I) kept at $278 \mathrm{~K}$.

\section{Crystal data}

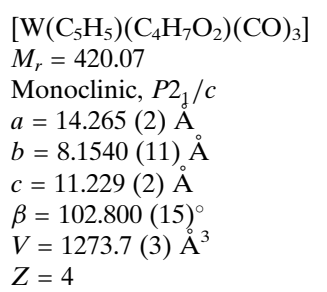

\author{
$D_{x}=2.191 \mathrm{Mg} \mathrm{m}^{-3}$ \\ Mo $K \alpha$ radiation \\ Cell parameters from 25 \\ reflections \\ $\theta=11.8-12.2^{\circ}$ \\ $\mu=9.08 \mathrm{~mm}^{-1}$ \\ $T=295$ (2) K \\ Rhomb, yellow \\ $0.40 \times 0.30 \times 0.30 \mathrm{~mm}$
}

\author{
Data collection \\ Enraf-Nonius CAD-4 \\ diffractometer \\ $\omega-2 \theta$ scans \\ Absorption correction: refined from \\ $\triangle F(D I F A B S$; Walker \& Stuart, \\ 1983) \\ $T_{\min }=0.011, T_{\max }=0.066$ \\ 5154 measured reflections \\ 2241 independent reflections
2053 reflections with $I>2 \sigma(I)$
$R_{\text {int }}=0.035$
$\theta_{\text {max }}=25.0^{\circ}$
$h=-16 \rightarrow 16$
$k=-1 \rightarrow 9$
$l=-13 \rightarrow 13$
3 standard reflections
frequency: $120 \mathrm{~min}$
intensity decay: $7 \%$ \\ Refinement \\ Refinement on $F^{2}$ \\ $R\left[F^{2}>2 \sigma\left(F^{2}\right)\right]=0.026$ \\ $w R\left(F^{2}\right)=0.072$ \\ $S=1.08$ \\ 2241 reflections \\ 164 parameters

$$
\begin{gathered}
w=1 /\left[\sigma^{2}\left(F_{o}^{2}\right)+(0.0482 P)^{2}\right. \\
\quad+0.7777 P] \\
\text { where } P=\left(F_{o}^{2}+2 F_{c}^{2}\right) / 3 \\
(\Delta / \sigma)_{\max }=0.001 \\
\Delta \rho_{\max }=1.00 \AA^{-3} \\
\Delta \rho_{\min }=-1.17 \mathrm{e}^{-3}
\end{gathered}
$$$$
\Delta \rho_{\max }=1.00{\mathrm{e} \AA^{-3}}^{-3}
$$

$\mathrm{H}$-atom parameters constrained

Table 1

Selected geometric parameters $\left(\AA{ }^{\circ}\right)$.

\begin{tabular}{lrlr}
\hline $\mathrm{W} 1-C g 1$ & $2.012(2)$ & $\mathrm{O} 3-\mathrm{C} 3$ & $1.135(5)$ \\
$\mathrm{W} 1-\mathrm{C} 1$ & $1.975(5)$ & $\mathrm{O} 4-\mathrm{C} 7$ & $1.305(5)$ \\
$\mathrm{W} 1-\mathrm{C} 2$ & $1.986(5)$ & $\mathrm{O} 5-\mathrm{C} 7$ & $1.211(5)$ \\
$\mathrm{W} 1-\mathrm{C} 3$ & $1.974(5)$ & $\mathrm{C} 4-\mathrm{C} 5$ & $1.509(6)$ \\
$\mathrm{W} 1-\mathrm{C} 4$ & $2.319(4)$ & $\mathrm{C} 5-\mathrm{C} 6$ & $1.535(5)$ \\
$\mathrm{O} 1-\mathrm{C} 1$ & $1.147(6)$ & $\mathrm{C} 6-\mathrm{C} 7$ & $1.494(6)$ \\
$\mathrm{O} 2-\mathrm{C} 2$ & $1.143(6)$ & & \\
$C g 1-\mathrm{W} 1-\mathrm{C} 1$ & $129.02(15)$ & $\mathrm{C} 1-\mathrm{W} 1-\mathrm{C} 3$ & $106.84(18)$ \\
$C g 1-\mathrm{W} 1-\mathrm{C} 2$ & $118.89(18)$ & $\mathrm{C} 1-\mathrm{W} 1-\mathrm{C} 4$ & $75.15(16)$ \\
$C g 1-\mathrm{W} 1-\mathrm{C} 3$ & $123.62(16)$ & $\mathrm{C} 2-\mathrm{W} 1-\mathrm{C} 3$ & $78.1(2)$ \\
$C g 1-\mathrm{W} 1-\mathrm{C} 4$ & $110.12(13)$ & $\mathrm{C} 2-\mathrm{W} 1-\mathrm{C} 4$ & $130.95(19)$ \\
$\mathrm{C} 1-\mathrm{W} 1-\mathrm{C} 2$ & $75.58(19)$ & $\mathrm{C} 3-\mathrm{W} 1-\mathrm{C} 4$ & $73.91(17)$ \\
& & & \\
$\mathrm{C} 1-\mathrm{W} 1-\mathrm{C} 4-\mathrm{C} 5$ & $50.8(3)$ & $\mathrm{W} 1-\mathrm{C} 4-\mathrm{C} 5-\mathrm{C} 6$ & $167.4(3)$ \\
$\mathrm{C} 2-\mathrm{W} 1-\mathrm{C} 4-\mathrm{C} 5$ & $106.0(4)$ & $\mathrm{C} 4-\mathrm{C} 5-\mathrm{C} 6-\mathrm{O} 4$ & $-67.5(6)$ \\
$\mathrm{C} 3-\mathrm{W} 1-\mathrm{C} 4-\mathrm{C} 5$ & $163.7(4)$ & $\mathrm{C} 4-\mathrm{C} 5-\mathrm{C} 6-\mathrm{O} 5$ & $-82.2(3)$ \\
\hline
\end{tabular}

Note: $C g 1$ is the centroid of the cyclopentadienyl ring.

Table 2

Hydrogen-bonding geometry $\left(\AA{ }^{\circ}\right)$.

\begin{tabular}{lllll}
\hline$D-\mathrm{H} \cdots A$ & $D-\mathrm{H}$ & $\mathrm{H} \cdots A$ & $D \cdots A$ & $D-\mathrm{H} \cdots A$ \\
\hline $\mathrm{O} 4-\mathrm{H} 4 \cdots \mathrm{O}^{\mathrm{i}}$ & 0.82 & 1.84 & $2.653(5)$ & 169
\end{tabular}

Symmetry code: (i) $1-x, 1-y, 1-z$.

Presented here is a rerefinement, after suitable transformation of the unit-cell parameters and the atomic coordinates and reindexing of the intensity data, of a structure previously solved and fully refined in the space group $P \overline{1}$. The need for the rerefinement was clearly indicated by a checkCIF level A alert and the form it should take was revealed by recourse to the $A D D S Y M$ routine of PLATON (Spek, 2003). Close examination of a drawing of the content of the original, supposedly triclinic, unit cell fully confirmed the $A D D S Y M$ findings. Further support for the rerefinement reported here is the improvement in $R[I>2 \sigma(I)]$ from 0.031 for the triclinic model to the value of 0.026 for the present refinement (with the number of refined parameters now half that of the triclinic refinement). In the final stages of the present refinement, $\mathrm{H}$ atoms were placed in calculated positions, with $X-\mathrm{H}=0.82,0.93$ and $0.97 \AA$ for hydroxyl, cyclopentadienyl and methylene $\mathrm{H}$ atoms, respectively, and refined using a riding model, with $U_{\text {iso }}(\mathrm{H})=1.5 U_{\text {eq }}(\mathrm{O})$ or $1.2 U_{\text {eq }}(\mathrm{C})$, as appropriate for the nature of $X$. The position of the hydroxyl group in terms of its rotation about 
the $\mathrm{C}-\mathrm{O}$ bond was also refined. The highest residual electrondensity peak is $0.99 \AA$ from atom W1.The deepest residual electrondensity hole lies $0.69 \AA$ from atom W1.

Data collection: $C A D-4 / P C$ (Enraf-Nonius, 1994); cell refinement: $C A D-4 / P C$; data reduction: XCAD4 (Harms \& Wocadlo, 1995); program(s) used to solve structure: SHELXS97 (Sheldrick, 1997); program(s) used to refine structure: SHELXL97 (Sheldrick, 1997); molecular graphics: ORTEP-3 for Windows (Farrugia, 1997); software used to prepare material for publication: SHELXL97 and PLATON (Spek, 2003).

We thank O. Munro for collecting the intensity data. We further thank the DAAD (Germany), NRF (South Africa) and the University of KwaZulu-Natal for support.

\section{References}

Ariyaratne, J. K. P., Bierrum, A. M., Green, M. L. H., Ishaq, M. \& Prout, C. K. (1969). J. Chem. Soc. A, pp. 1309-1321.

Enraf-Nonius (1994). CAD-4/PC. Enraf-Nonius, Delft, The Netherlands.

Farrugia, L. J. (1997). J. Appl. Cryst. 30, 565.

Friedrich, H. B., Howie, R. A., Laing, M. \& Onani, M. O. (2004). J. Organomet. Chem. 689, 181-193.

Harms, K. \& Wocadlo, S. (1995). XCAD4. University of Marburg, Germany.

Onani, M. O. (2002). PhD thesis, University of KwaZulu-Natal, Durban, South Africa.

Sheldrick, G. M. (1997). SHELXS97 and SHELXL97. University of Göttingen, Germany.

Spek, A. L. (2003). J. Appl. Cryst. 36, 7-13.

Walker, N. \& Stuart, D. (1983). Acta Cryst. A39, 158-166. 


\title{
supporting information
}

Acta Cryst. (2004). E60, m1641-m1643 [https://doi.org/10.1107/S1600536804025371]

\section{Tricarbonyl(3-carboxypropyl) ( $\eta^{5}$-cyclopentadienyl)tungsten(II)}

\author{
Holger B. Friedrich, R. Alan Howie and Martin O. Onani
}

Tricarbonyl(3-carboxypropyl)( $\eta^{5}$-cyclopentadienyl)tungsten(II)

Crystal data

$\left[\mathrm{W}\left(\mathrm{C}_{5} \mathrm{H}_{5}\right)\left(\mathrm{C}_{4} \mathrm{H}_{7} \mathrm{O}_{2}\right)(\mathrm{CO})_{3}\right]$

$F(000)=792$

$M_{r}=420.07$

Monoclinic, $P 2_{1} / c$

Hall symbol: P -2ybc

$a=14.265(2) \AA$

$b=8.1540$ (11) $\AA$

$c=11.229(2) \AA$

$\beta=102.800(15)^{\circ}$

$D_{\mathrm{x}}=2.191 \mathrm{Mg} \mathrm{m}^{-3}$

Mo $K \alpha$ radiation, $\lambda=0.71073 \AA$

Cell parameters from 25 reflections

$\theta=11.8-12.2^{\circ}$

$\mu=9.08 \mathrm{~mm}^{-1}$

$T=295 \mathrm{~K}$

$V=1273.7(3) \AA^{3}$

Rhomb, yellow

$Z=4$

$0.40 \times 0.30 \times 0.30 \mathrm{~mm}$

\section{Data collection}

Enraf-Nonius CAD-4 diffractometer

Radiation source: fine-focus sealed tube

2241 independent reflections

Graphite monochromator

$\omega-2 \theta$ scans

Absorption correction: part of the refinement model $(\Delta F)$

(DIFABS; Walker \& Stuart, 1983)

$T_{\min }=0.011, T_{\max }=0.066$

5154 measured reflections 2053 reflections with $I>2 \sigma(I)$

$R_{\text {int }}=0.035$

$\theta_{\max }=25.0^{\circ}, \theta_{\min }=2.9^{\circ}$

$h=-16 \rightarrow 16$

$k=-1 \rightarrow 9$

$l=-13 \rightarrow 13$

3 standard reflections every $120 \mathrm{~min}$

intensity decay: $7 \%$

Refinement

Refinement on $F^{2}$

Least-squares matrix: full

$R\left[F^{2}>2 \sigma\left(F^{2}\right)\right]=0.026$

$w R\left(F^{2}\right)=0.072$

$S=1.08$

2241 reflections

164 parameters

0 restraints

Primary atom site location: structure-invariant direct methods

Secondary atom site location: difference Fourier map

Hydrogen site location: inferred from neighbouring sites

$\mathrm{H}$-atom parameters constrained

$w=1 /\left[\sigma^{2}\left(F_{\mathrm{o}}^{2}\right)+(0.0482 P)^{2}+0.7777 P\right]$ where $P=\left(F_{\mathrm{o}}^{2}+2 F_{\mathrm{c}}^{2}\right) / 3$

$(\Delta / \sigma)_{\max }=0.001$

$\Delta \rho_{\max }=1.00 \mathrm{e} \AA^{-3}$

$\Delta \rho_{\min }=-1.17 \mathrm{e} \AA^{-3}$ 


\section{Special details}

Geometry. All e.s.d.'s (except the e.s.d. in the dihedral angle between two l.s. planes) are estimated using the full covariance matrix. The cell e.s.d.'s are taken into account individually in the estimation of e.s.d.'s in distances, angles and torsion angles; correlations between e.s.d.'s in cell parameters are only used when they are defined by crystal symmetry. An approximate (isotropic) treatment of cell e.s.d.'s is used for estimating e.s.d.'s involving l.s. planes. Distance (1 entry) and angles ( 4 entries) involving $C g 1$ (the centroid of the cyclopentadienyl ring) have been entered by hand into the relevant sections of the cif for ease of reference.

Least-squares planes $(x, y, z$ in crystal coordinates) and deviations from them $(*$ indicates atom used to define plane) $4.4564(0.0323) x+4.7000(0.0174) y+7.4911(0.0205) z=8.9230(0.0227)$

* $-0.0042(0.0029) \mathrm{C} 8 * 0.0026(0.0030) \mathrm{C} 9 * 0.0001(0.0031) \mathrm{C} 10 *-0.0027(0.0032) \mathrm{C} 11 * 0.0043(0.0030) \mathrm{C} 12$ $2.0105(0.0021) \mathrm{W} 1-3.2724(0.0062) \mathrm{C} 1-2.9105(0.0073) \mathrm{C} 2-3.0909(0.0066) \mathrm{C} 3$

Rms deviation of fitted atoms $=0.0032$

$2.8996(0.0447) x+4.0968(0.0144) y+8.6965(0.0214) z=4.3963(0.0356)$

Angle to previous plane (with approximate e.s.d.) $=9.01(0.38)$

* $0.0000(0.0000) \mathrm{C} 1 * 0.0000(0.0000) \mathrm{C} 2 * 0.0000(0.0000) \mathrm{C} 31.1472(0.0028) \mathrm{W} 13.1471(0.0060) \mathrm{C} 83.3253$

(0.0063) C9 $3.2498(0.0064)$ C10 $3.0270(0.0066)$ C11 2.9704 (0.0066) C12 - 0.6372 (0.0067) O1 - 0.6798 (0.0093) O2 $0.6125(0.0071) \mathrm{O} 3$

Rms deviation of fitted atoms $=0.0000$

$3.1651(0.0224) x+4.0974(0.0075) y+8.5980(0.0115) z=3.9634(0.0183)$

Angle to previous plane (with approximate e.s.d.) $=1.10(0.31)$

$* 0.0000(0.0000) \mathrm{O} 1 * 0.0000(0.0000) \mathrm{O} 2 * 0.0000(0.0000) \mathrm{O} 3$

Rms deviation of fitted atoms $=0.0000$

Refinement. Refinement of $F^{2}$ against ALL reflections. The weighted $R$-factor $w R$ and goodness of fit $S$ are based on $F^{2}$, conventional $R$-factors $R$ are based on $F$, with $F$ set to zero for negative $F^{2}$. The threshold expression of $F^{2}>\sigma\left(F^{2}\right)$ is used only for calculating $R$-factors(gt) $e t c$. and is not relevant to the choice of reflections for refinement. $R$-factors based on $F^{2}$ are statistically about twice as large as those based on $F$, and $R$ - factors based on ALL data will be even larger.

$\mathrm{H}$ atoms placed in calculated positions with $X-\mathrm{H} 0.82,0.93$ and $0.97 \mathrm{~A}$ for hydroxyl, cyclopentadienyl and methylene $\mathrm{H}$, respectively, and refined with a riding model with $U_{\text {iso }}(\mathrm{H})=1.5 \mathrm{Ueq}(\mathrm{O})$ or $1.2 \mathrm{Ueq}(\mathrm{C})$ as appropriate for the nature of $X$. The position of the hydroxyl group in terms of its rotation about the $\mathrm{C}-\mathrm{O}$ bond was also refined.

Fractional atomic coordinates and isotropic or equivalent isotropic displacement parameters $\left(\AA^{2}\right)$

\begin{tabular}{lllll}
\hline & $x$ & $y$ & $z$ & $U_{\text {iso }} * / U_{\text {eq }}$ \\
\hline W1 & $0.800314(12)$ & $0.48662(2)$ & $0.141360(15)$ & $0.03491(11)$ \\
O1 & $0.8107(2)$ & $0.1070(5)$ & $0.1116(3)$ & $0.0607(9)$ \\
O2 & $0.9002(3)$ & $0.4342(6)$ & $-0.0773(4)$ & $0.0759(11)$ \\
O3 & $0.6469(3)$ & $0.6263(5)$ & $-0.0757(3)$ & $0.0655(9)$ \\
O4 & $0.4419(2)$ & $0.3506(5)$ & $0.3892(3)$ & $0.0621(9)$ \\
H4 & 0.4325 & 0.4221 & 0.4365 & $0.093^{*}$ \\
O5 & $0.5900(3)$ & $0.4493(5)$ & $0.4377(3)$ & $0.0567(8)$ \\
C1 & $0.8043(3)$ & $0.2463(6)$ & $0.1213(4)$ & $0.0394(9)$ \\
C2 & $0.8645(4)$ & $0.4546(7)$ & $0.0031(5)$ & $0.0523(12)$ \\
C3 & $0.7026(3)$ & $0.5712(6)$ & $0.0022(4)$ & $0.0465(10)$ \\
C4 & $0.6532(3)$ & $0.3944(5)$ & $0.1705(4)$ & $0.0408(9)$ \\
H4A & 0.6202 & 0.4871 & 0.1966 & $0.049 *$ \\
H4B & 0.6154 & 0.3596 & 0.0918 & $0.049 *$ \\
C5 & $0.6527(3)$ & $0.2565(5)$ & $0.2599(4)$ & $0.0439(10)$ \\
H5A & 0.7003 & 0.2791 & 0.3343 & $0.053^{*}$ \\
H5B & 0.6710 & 0.1555 & 0.2254 & $0.053^{*}$ \\
C6 & $0.5543(3)$ & $0.2328(5)$ & $0.2916(4)$ & $0.0471(10)$ \\
H6A & 0.5050 & 0.2374 & 0.2167 & $0.057^{*}$
\end{tabular}




\begin{tabular}{lllll} 
H6B & 0.5522 & 0.1243 & 0.3263 & $0.057^{*}$ \\
C7 & $0.5312(3)$ & $0.3564(5)$ & $0.3790(4)$ & $0.0425(9)$ \\
C8 & $0.8027(4)$ & $0.7247(6)$ & $0.2584(4)$ & $0.0564(12)$ \\
H8 & 0.7539 & 0.8020 & 0.2378 & $0.068^{*}$ \\
C9 & $0.8052(3)$ & $0.5954(6)$ & $0.3390(4)$ & $0.0512(11)$ \\
H9 & 0.7585 & 0.5715 & 0.3825 & $0.061^{*}$ \\
C10 & $0.8902(5)$ & $0.5065(5)$ & $0.3438(6)$ & $0.0542(15)$ \\
H10 & 0.9095 & 0.4135 & 0.3907 & $0.065^{*}$ \\
C11 & $0.9411(3)$ & $0.5829(7)$ & $0.2652(5)$ & $0.0563(12)$ \\
H11 & 0.9998 & 0.5488 & 0.2510 & $0.068^{*}$ \\
C12 & $0.8885(4)$ & $0.7185(6)$ & $0.2124(5)$ & $0.0584(12)$ \\
H12 & 0.9059 & 0.7914 & 0.1574 & $0.070^{*}$ \\
\hline
\end{tabular}

Atomic displacement parameters $\left(\AA^{2}\right)$

\begin{tabular}{lllllll}
\hline & $U^{11}$ & $U^{22}$ & $U^{33}$ & $U^{12}$ & $U^{13}$ & $U^{23}$ \\
\hline W1 & $0.03475(17)$ & $0.03509(14)$ & $0.03487(16)$ & $0.00048(5)$ & $0.00766(11)$ & $-0.00016(5)$ \\
O1 & $0.063(2)$ & $0.047(2)$ & $0.072(2)$ & $0.0105(16)$ & $0.0141(17)$ & $-0.0033(17)$ \\
O2 & $0.078(3)$ & $0.096(3)$ & $0.066(2)$ & $-0.004(3)$ & $0.044(2)$ & $-0.008(2)$ \\
O3 & $0.064(2)$ & $0.071(2)$ & $0.055(2)$ & $0.0085(19)$ & $-0.0007(17)$ & $0.0166(18)$ \\
O4 & $0.0410(18)$ & $0.081(3)$ & $0.068(2)$ & $-0.0133(17)$ & $0.0219(16)$ & $-0.0192(19)$ \\
O5 & $0.0394(19)$ & $0.072(2)$ & $0.060(2)$ & $-0.0114(18)$ & $0.0157(17)$ & $-0.016(2)$ \\
C1 & $0.031(2)$ & $0.040(3)$ & $0.046(2)$ & $0.0026(16)$ & $0.0054(17)$ & $-0.0030(17)$ \\
C2 & $0.052(3)$ & $0.054(3)$ & $0.053(3)$ & $-0.003(2)$ & $0.017(3)$ & $-0.001(2)$ \\
C3 & $0.053(3)$ & $0.043(2)$ & $0.045(2)$ & $0.000(2)$ & $0.014(2)$ & $0.001(2)$ \\
C4 & $0.035(2)$ & $0.049(2)$ & $0.038(2)$ & $-0.0004(18)$ & $0.0072(17)$ & $0.0001(18)$ \\
C5 & $0.042(2)$ & $0.041(2)$ & $0.050(2)$ & $0.0016(18)$ & $0.014(2)$ & $0.0023(19)$ \\
C6 & $0.045(2)$ & $0.043(2)$ & $0.053(2)$ & $-0.0043(19)$ & $0.012(2)$ & $0.0041(19)$ \\
C7 & $0.038(2)$ & $0.048(2)$ & $0.042(2)$ & $-0.0002(19)$ & $0.0117(18)$ & $0.0103(19)$ \\
C8 & $0.063(3)$ & $0.041(2)$ & $0.061(3)$ & $0.001(2)$ & $0.004(2)$ & $-0.012(2)$ \\
C9 & $0.054(3)$ & $0.057(3)$ & $0.043(2)$ & $-0.007(2)$ & $0.011(2)$ & $-0.014(2)$ \\
C10 & $0.059(4)$ & $0.057(3)$ & $0.042(3)$ & $-0.001(2)$ & $0.001(3)$ & $-0.0020(18)$ \\
C11 & $0.039(2)$ & $0.070(3)$ & $0.056(3)$ & $-0.010(2)$ & $0.000(2)$ & $-0.008(3)$ \\
C12 & $0.062(3)$ & $0.046(3)$ & $0.064(3)$ & $-0.022(2)$ & $0.006(2)$ & $-0.004(2)$ \\
& & & & & & \\
\hline
\end{tabular}

Geometric parameters $\left(\AA,{ }^{o}\right)$

\begin{tabular}{llll}
\hline $\mathrm{W} 1-\mathrm{Cg} 1$ & $2.012(2)$ & $\mathrm{C} 4-\mathrm{H} 4 \mathrm{~B}$ & 0.9700 \\
$\mathrm{~W} 1-\mathrm{C} 1$ & $1.975(5)$ & $\mathrm{C} 5-\mathrm{C} 6$ & $1.535(5)$ \\
$\mathrm{W} 1-\mathrm{C} 2$ & $1.986(5)$ & $\mathrm{C} 5-\mathrm{H} 5 \mathrm{~A}$ & 0.9700 \\
$\mathrm{~W} 1-\mathrm{C} 3$ & $1.974(5)$ & $\mathrm{C} 5-\mathrm{H} 5 \mathrm{~B}$ & 0.9700 \\
$\mathrm{~W} 1-\mathrm{C} 11$ & $2.312(4)$ & $\mathrm{C} 6-\mathrm{C} 7$ & $1.494(6)$ \\
$\mathrm{W} 1-\mathrm{C} 12$ & $2.313(4)$ & $\mathrm{C} 6-\mathrm{H} 6 \mathrm{~A}$ & 0.9700 \\
$\mathrm{~W} 1-\mathrm{C} 4$ & $2.319(4)$ & $\mathrm{C} 6-\mathrm{H} 6 \mathrm{~B}$ & 0.9700 \\
$\mathrm{~W} 1-\mathrm{C} 8$ & $2.340(4)$ & $\mathrm{C} 8-\mathrm{C} 9$ & $1.385(7)$ \\
$\mathrm{W} 1-\mathrm{C} 10$ & $2.355(6)$ & $\mathrm{C} 8-\mathrm{C} 12$ & $1.431(7)$ \\
$\mathrm{W} 1-\mathrm{C} 9$ & $2.376(4)$ & $\mathrm{C} 8-\mathrm{H} 8$ & 0.9300 \\
$\mathrm{O} 1-\mathrm{C} 1$ & $1.147(6)$ & $\mathrm{C} 9-\mathrm{C} 10$ & $1.403(8)$
\end{tabular}




\begin{tabular}{|c|c|c|c|}
\hline $\mathrm{O} 2-\mathrm{C} 2$ & $1.143(6)$ & C9-H9 & 0.9300 \\
\hline $\mathrm{O} 3-\mathrm{C} 3$ & $1.135(5)$ & $\mathrm{C} 10-\mathrm{C} 11$ & $1.406(8)$ \\
\hline $\mathrm{O} 4-\mathrm{C} 7$ & $1.305(5)$ & $\mathrm{C} 10-\mathrm{H} 10$ & 0.9300 \\
\hline $\mathrm{O} 4-\mathrm{H} 4$ & 0.8200 & $\mathrm{C} 11-\mathrm{C} 12$ & $1.392(7)$ \\
\hline $\mathrm{O} 5-\mathrm{C} 7$ & $1.211(5)$ & $\mathrm{C} 11-\mathrm{H} 11$ & 0.9300 \\
\hline $\mathrm{C} 4-\mathrm{C} 5$ & $1.509(6)$ & $\mathrm{C} 12-\mathrm{H} 12$ & 0.9300 \\
\hline $\mathrm{C} 4-\mathrm{H} 4 \mathrm{~A}$ & 0.9700 & & \\
\hline $\mathrm{Cg} 1-\mathrm{W} 1-\mathrm{C} 1$ & $129.02(15)$ & $\mathrm{W} 1-\mathrm{C} 4-\mathrm{H} 4 \mathrm{~B}$ & 107.7 \\
\hline $\mathrm{Cg} 1-\mathrm{W} 1-\mathrm{C} 2$ & $118.89(18)$ & $\mathrm{H} 4 \mathrm{~A}-\mathrm{C} 4-\mathrm{H} 4 \mathrm{~B}$ & 107.1 \\
\hline $\mathrm{Cg} 1-\mathrm{W} 1-\mathrm{C} 3$ & $123.62(16)$ & $\mathrm{C} 4-\mathrm{C} 5-\mathrm{C} 6$ & $112.8(3)$ \\
\hline $\mathrm{Cg} 1-\mathrm{W} 1-\mathrm{C} 4$ & $110.12(13)$ & $\mathrm{C} 4-\mathrm{C} 5-\mathrm{H} 5 \mathrm{~A}$ & 109.0 \\
\hline $\mathrm{C} 1-\mathrm{W} 1-\mathrm{C} 2$ & $75.58(19)$ & $\mathrm{C} 6-\mathrm{C} 5-\mathrm{H} 5 \mathrm{~A}$ & 109.0 \\
\hline $\mathrm{C} 1-\mathrm{W} 1-\mathrm{C} 3$ & $106.84(18)$ & $\mathrm{C} 4-\mathrm{C} 5-\mathrm{H} 5 \mathrm{~B}$ & 109.0 \\
\hline $\mathrm{C} 1-\mathrm{W} 1-\mathrm{C} 4$ & $75.15(16)$ & $\mathrm{C} 6-\mathrm{C} 5-\mathrm{H} 5 \mathrm{~B}$ & 109.0 \\
\hline $\mathrm{C} 2-\mathrm{W} 1-\mathrm{C} 3$ & $78.1(2)$ & $\mathrm{H} 5 \mathrm{~A}-\mathrm{C} 5-\mathrm{H} 5 \mathrm{~B}$ & 107.8 \\
\hline $\mathrm{C} 2-\mathrm{W} 1-\mathrm{C} 4$ & $130.95(19)$ & $\mathrm{C} 7-\mathrm{C} 6-\mathrm{C} 5$ & 114.5 (4) \\
\hline $\mathrm{C} 3-\mathrm{W} 1-\mathrm{C} 4$ & $73.91(17)$ & $\mathrm{C} 7-\mathrm{C} 6-\mathrm{H} 6 \mathrm{~A}$ & 108.6 \\
\hline $\mathrm{C} 3-\mathrm{W} 1-\mathrm{C} 11$ & $136.4(2)$ & $\mathrm{C} 5-\mathrm{C} 6-\mathrm{H} 6 \mathrm{~A}$ & 108.6 \\
\hline $\mathrm{C} 1-\mathrm{W} 1-\mathrm{C} 11$ & $111.26(19)$ & $\mathrm{C} 7-\mathrm{C} 6-\mathrm{H} 6 \mathrm{~B}$ & 108.6 \\
\hline $\mathrm{C} 2-\mathrm{W} 1-\mathrm{C} 11$ & $91.5(2)$ & $\mathrm{C} 5-\mathrm{C} 6-\mathrm{H} 6 \mathrm{~B}$ & 108.6 \\
\hline $\mathrm{C} 3-\mathrm{W} 1-\mathrm{C} 12$ & $102.8(2)$ & $\mathrm{H} 6 \mathrm{~A}-\mathrm{C} 6-\mathrm{H} 6 \mathrm{~B}$ & 107.6 \\
\hline $\mathrm{C} 1-\mathrm{W} 1-\mathrm{C} 12$ & $145.43(17)$ & $\mathrm{O} 5-\mathrm{C} 7-\mathrm{O} 4$ & $123.2(4)$ \\
\hline $\mathrm{C} 2-\mathrm{W} 1-\mathrm{C} 12$ & $93.8(2)$ & $\mathrm{O} 5-\mathrm{C} 7-\mathrm{C} 6$ & $123.5(4)$ \\
\hline $\mathrm{C} 11-\mathrm{W} 1-\mathrm{C} 12$ & 35.04 (19) & $\mathrm{O} 4-\mathrm{C} 7-\mathrm{C} 6$ & $113.3(4)$ \\
\hline $\mathrm{C} 11-\mathrm{W} 1-\mathrm{C} 4$ & $135.87(17)$ & $\mathrm{C} 9-\mathrm{C} 8-\mathrm{C} 12$ & $108.0(5)$ \\
\hline $\mathrm{C} 12-\mathrm{W} 1-\mathrm{C} 4$ & $131.05(18)$ & $\mathrm{C} 9-\mathrm{C} 8-\mathrm{W} 1$ & $74.4(3)$ \\
\hline $\mathrm{C} 3-\mathrm{W} 1-\mathrm{C} 8$ & $94.28(18)$ & $\mathrm{C} 12-\mathrm{C} 8-\mathrm{W} 1$ & $71.1(3)$ \\
\hline $\mathrm{C} 1-\mathrm{W} 1-\mathrm{C} 8$ & $152.86(17)$ & $\mathrm{C} 9-\mathrm{C} 8-\mathrm{H} 8$ & 126.0 \\
\hline $\mathrm{C} 2-\mathrm{W} 1-\mathrm{C} 8$ & $126.6(2)$ & $\mathrm{C} 12-\mathrm{C} 8-\mathrm{H} 8$ & 126.0 \\
\hline $\mathrm{C} 11-\mathrm{W} 1-\mathrm{C} 8$ & $58.51(19)$ & $\mathrm{W} 1-\mathrm{C} 8-\mathrm{H} 8$ & 120.4 \\
\hline $\mathrm{C} 12-\mathrm{W} 1-\mathrm{C} 8$ & $35.81(18)$ & $\mathrm{C} 8-\mathrm{C} 9-\mathrm{C} 10$ & $108.3(5)$ \\
\hline $\mathrm{C} 4-\mathrm{W} 1-\mathrm{C} 8$ & 95.24 (17) & $\mathrm{C} 8-\mathrm{C} 9-\mathrm{W} 1$ & $71.5(3)$ \\
\hline $\mathrm{C} 3-\mathrm{W} 1-\mathrm{C} 10$ & $151.43(19)$ & $\mathrm{C} 10-\mathrm{C} 9-\mathrm{W} 1$ & $71.9(3)$ \\
\hline $\mathrm{C} 1-\mathrm{W} 1-\mathrm{C} 10$ & $98.92(16)$ & $\mathrm{C} 8-\mathrm{C} 9-\mathrm{H} 9$ & 125.8 \\
\hline $\mathrm{C} 2-\mathrm{W} 1-\mathrm{C} 10$ & $121.1(2)$ & $\mathrm{C} 10-\mathrm{C} 9-\mathrm{H} 9$ & 125.8 \\
\hline $\mathrm{C} 11-\mathrm{W} 1-\mathrm{C} 10$ & $35.1(2)$ & $\mathrm{W} 1-\mathrm{C} 9-\mathrm{H} 9$ & 122.4 \\
\hline $\mathrm{C} 12-\mathrm{W} 1-\mathrm{C} 10$ & $58.17(18)$ & $\mathrm{C} 9-\mathrm{C} 10-\mathrm{C} 11$ & $108.0(5)$ \\
\hline $\mathrm{C} 4-\mathrm{W} 1-\mathrm{C} 10$ & $101.69(19)$ & $\mathrm{C} 9-\mathrm{C} 10-\mathrm{W} 1$ & $73.6(3)$ \\
\hline $\mathrm{C} 8-\mathrm{W} 1-\mathrm{C} 10$ & $57.57(17)$ & $\mathrm{C} 11-\mathrm{C} 10-\mathrm{W} 1$ & $70.8(3)$ \\
\hline $\mathrm{C} 3-\mathrm{W} 1-\mathrm{C} 9$ & $118.59(18)$ & $\mathrm{C} 9-\mathrm{C} 10-\mathrm{H} 10$ & 126.0 \\
\hline $\mathrm{C} 1-\mathrm{W} 1-\mathrm{C} 9$ & $118.74(17)$ & $\mathrm{C} 11-\mathrm{C} 10-\mathrm{H} 10$ & 126.0 \\
\hline $\mathrm{C} 2-\mathrm{W} 1-\mathrm{C} 9$ & $148.82(19)$ & $\mathrm{W} 1-\mathrm{C} 10-\mathrm{H} 10$ & 121.3 \\
\hline $\mathrm{C} 11-\mathrm{W} 1-\mathrm{C} 9$ & $57.98(17)$ & $\mathrm{C} 12-\mathrm{C} 11-\mathrm{C} 10$ & $108.4(5)$ \\
\hline $\mathrm{C} 12-\mathrm{W} 1-\mathrm{C} 9$ & $58.12(18)$ & $\mathrm{C} 12-\mathrm{C} 11-\mathrm{W} 1$ & $72.5(3)$ \\
\hline $\mathrm{C} 4-\mathrm{W} 1-\mathrm{C} 9$ & $80.20(16)$ & $\mathrm{C} 10-\mathrm{C} 11-\mathrm{W} 1$ & $74.1(3)$ \\
\hline $\mathrm{C} 8-\mathrm{W} 1-\mathrm{C} 9$ & 34.14 (17) & $\mathrm{C} 12-\mathrm{C} 11-\mathrm{H} 11$ & 125.8 \\
\hline $\mathrm{C} 10-\mathrm{W} 1-\mathrm{C} 9$ & $34.51(19)$ & $\mathrm{C} 10-\mathrm{C} 11-\mathrm{H} 11$ & 125.8 \\
\hline
\end{tabular}




$\begin{array}{llll}\mathrm{C} 7-\mathrm{O} 4-\mathrm{H} 4 & 109.5 & \mathrm{~W} 1-\mathrm{C} 11-\mathrm{H} 11 & 119.4 \\ \mathrm{O} 1-\mathrm{C} 1-\mathrm{W} 1 & 177.1(4) & \mathrm{C} 11-\mathrm{C} 12-\mathrm{C} 8 & 107.3(5) \\ \mathrm{O} 2-\mathrm{C} 2-\mathrm{W} 1 & 178.8(5) & \mathrm{C} 11-\mathrm{C} 12-\mathrm{W} 1 & 72.5(3) \\ \mathrm{O} 3-\mathrm{C} 3-\mathrm{W} 1 & 177.1(4) & \mathrm{C} 8-\mathrm{C} 12-\mathrm{W} 1 & 73.1(3) \\ \mathrm{C} 5-\mathrm{C} 4-\mathrm{W} 1 & 118.3(3) & \mathrm{C} 11-\mathrm{C} 12-\mathrm{H} 12 & 126.4 \\ \mathrm{C} 5-\mathrm{C} 4-\mathrm{H} 4 \mathrm{~A} & 107.7 & \mathrm{C} 8-\mathrm{C} 12-\mathrm{H} 12 & 126.4 \\ \mathrm{~W} 1-\mathrm{C} 4-\mathrm{H} 4 \mathrm{~A} & 107.7 & \mathrm{~W} 1-\mathrm{C} 12-\mathrm{H} 12 & 119.9 \\ \mathrm{C} 5-\mathrm{C} 4-\mathrm{H} 4 \mathrm{~B} & 107.7 & & \\ & & & 167.4(3) \\ \mathrm{C} 1-\mathrm{W} 1-\mathrm{C} 4-\mathrm{C} 5 & 50.8(3) & \mathrm{W} 1-\mathrm{C} 4-\mathrm{C} 5-\mathrm{C} 6 & -67.5(6) \\ \mathrm{C} 2-\mathrm{W} 1-\mathrm{C} 4-\mathrm{C} 5 & 106.0(4) & \mathrm{C} 4-\mathrm{C} 5-\mathrm{C} 6-\mathrm{O} 4 & -82.2(3) \\ \mathrm{C} 3-\mathrm{W} 1-\mathrm{C} 4-\mathrm{C} 5 & 163.7(4) & \mathrm{C} 4-\mathrm{C} 5-\mathrm{C} 6-\mathrm{O} 5 & \end{array}$

Hydrogen-bond geometry $\left(\AA,{ }^{\circ}\right)$

\begin{tabular}{lllll}
\hline$D-\mathrm{H} \cdots A$ & $D-\mathrm{H}$ & $\mathrm{H} \cdots A$ & $D \cdots A$ & $D-\mathrm{H} \cdots A$ \\
\hline $\mathrm{O} 4-\mathrm{H} 4 \cdots \mathrm{O}^{\mathrm{i}}$ & 0.82 & 1.84 & $2.653(5)$ & 169 \\
\hline
\end{tabular}

Symmetry code: (i) $-x+1,-y+1,-z+1$. 